\title{
A THREE-DIMENSIONAL RANDOM ISING MODEL: RESUMMATION OF FIVE-LOOP SERIES
}

\author{
V. Blavats'ka ${ }^{1}$, Yu. Holovatch ${ }^{1,2}$ \\ ${ }^{1}$ Institute for Condensed Matter Physics of the National Academy of Sciences of Ukraine, \\ 1 Svientsitskii Str., Lviv, UA-79011, Ukraine \\ ${ }^{2}$ Ivan Franko National University of Lviv, Department for Theoretical Physics, \\ 12 Drahomanov Str., Lviv, UA-79005, Ukraine
}

(Received August 30, 2001)

\begin{abstract}
We investigate the critical behaviour of a $d=3$-dimensional weakly diluted quenched Ising model, analyzing the series for the renormalization group functions at $d=3$ obtained in a minimal subtraction scheme. Formerly it was established [R. Folk, Yu. Holovatch, T. Yavorskii, Phys. Rev. B 61, $15114(2000)$ ] that the resummed renormalization group series possess an "optimal truncation" behaviour, provided that a Chisholm-Borel resummation technique is applied. This resulted in a conjecture that a four-loop approximation is a final one for the random Ising model renormalizationgroup functions in a $d=3$ minimal subtraction scheme. We apply the method of subsequent resummation, developed in the context of the $d=0$-dimensional random Ising model, discuss the convergence properties of the series and give the results of the critical exponents increasing the order of approximation to 5-loop level.
\end{abstract}

Key words: renormalization group, the critical exponents, asymptotic series.

PACS number(s): 61.43.-j, 05.10.Cc, 64.60.-i, 75.10.Hk

\section{INTRODUCTION}

An effect of the weak quenched disorder on the critical behaviour of the magnetic systems can be forecasted by means of Harris criterion [1]. It states that the addition of impurities to the system undergoing a second order phase transition causes changes in the critical behaviour only if the heat capacity critical exponent of the pure system $\alpha_{\text {pure }}>0$, i.e., the heat capacity diverges at the critical point. Examination of the different realizations of the general $m$-vector model of a magnet reveals that only pure Ising model (that corresponds to the $m=1$ case) is characterized by the value of $\alpha>0$ and, thus, is affected by the weakly quenched disorder at criticality.

Theoretical studies of the critical properties of the weakly diluted quenched Ising model (random Ising model - RIM) are based on the renormalization group (RG) approach [2]; here, the change of the universal properties is interpreted as a crossover from the "pure" fixed point present in the undiluted model towards the "random" one which characterizes the new critical behaviour of RIM. Although up to now the values of critical exponents of RIM have been estimated in numerous studies, the situation here is less satisfactory than in the pure case (see, e.g., [3] for a recent review).

Closely examining the critical behaviour of the model by means of RG approach reveals the problem in dealing with RG functions in the form of perturbation series with zero radius of convergence. A situation in which the nature of the divergence is such that the series is asymptotic is, at least in principle, controllable: in such a case a good estimate for the sum of the series is obtained by keeping a certain number of the first terms ("optimal truncation"). A self-consistent way to take into account the higher contributions requires an application of special resummation procedures [4]. Resumming a series, one should note that the precision of the obtained results depends on the resummation procedure. Thus, the analysis and an appropriate selection of the resummation procedure is crucial for obtaining reliable final results.

Concerning the function $f(g)$ in the form of power series

$$
f(g)=\sum_{k=0}^{\infty} a_{k} g^{k}
$$

the basic definition of its asymptotic nature is:

$$
\left|f(g)-\sum_{k=0}^{N} a_{k} g^{k}\right| \leq a_{k+1} g^{k+1}
$$

for fixed $N$ and small $g$.

In general, the nature of asymptotic series is such that the correct sum is uniformly approached until, after an optimum number of terms $N_{0}$ additional terms drive the sum away from the correct result, leading to a divergence $[4]$.

For the models with a simple $O(m)$-symmetry, in the large orders of perturbation theory the factorial growth of the RG functions coefficients has been established [5]:

$$
a_{k}=k !(-a)^{k} k^{b} c\left[1+O\left(\frac{1}{k}\right)\right], \quad k \rightarrow \infty
$$

the quantities $a$ and $b$ have been calculated in Ref. [6], the values of constant $c$ in Ref. [7]. The estimate (1) is a fundamental one since it proves that initial functions 


\section{BLAVATS'KA, YU. HOLOVATCH}

can be reconstituted from their asymptotic expansions by application of integral Borel transformation [8] in different modifications. Indeed, its application to the analytical calculations of the critical exponents of the pure Ising model leads to convergent results as the order of perturbation theory increases [9].

The RIM problem is addressed next. Notably, the corresponding $\mathrm{RG}$ equations are degenerated on the oneloop level, leading to the expansion in $\sqrt{\varepsilon}[10-12]$ instead of the familiar expansion in $\varepsilon=4-d$. This expansion was recently extended from the 3 -loop [13] to the 5-loop level [14] for the critical exponents. However, the nature of the $\sqrt{\varepsilon}$ expansion does not allow us to obtain reliable information at $d=3$, which can be considered as an indirect evidence for the non-asymptotic nature of the expansion. The fact that $\varepsilon$-expansion will not be able to give information on critical exponents in RIM was predicted already in Refs. [15]. There, studying the randomly diluted model in zero dimensions, it was shown that the non-Borel summable properties of the perturbation theory series are the direct consequence of the existence of Griffiths-like singularities [16] caused by the zeroes of the partition function of the pure model.

RG equations of the massive field theory at fixed $d$ [17] appeared to be the most efficient if the estimations for the $d=3$ case are to be made. The values of the critical exponents of the RIM were obtained, considering the resulting expansions as if they are asymptotic and applying appropriate resummation procedures increasing the order of the perturbation theory series from the 2-loop (2LA) [18] through 3-loop (3LA) [19],4-loop (4LA) [20], 5 -loop [21] to the record 6-loop approximation [22]. However, the familiar Chisholm-Borel resummation scheme applied to the successive orders of the perturbation theory series appears to encounter serious difficulties already on the 5-loop level [3]. This might point towards the possible non-asymptotic nature of RG series and to appearance of an "optimal truncation" regime for the resummed series.

The minimal subtraction RG scheme [23] is also appropriate for the $d=3$ renormalization and can be applied without $\varepsilon$-expansion [24]. In this scheme, the values of the RIM critical exponents were obtained recently in Ref. [25] up to 4-loop approximation. In 5 loop, however, no stable random fixed point was found, which led to conjecture that in this case the 4-loop approximation is the "optimal truncation" for the resummed perturbation theory series, similar as it holds in the nonresummed asymptotic series.

Recently, it was demonstrated analytically, that RG functions of the $d=0$ RIM are Borel-summable if the special technique - the so-called subsequent resummation - is exploited [27]. The application of this technique to the $d=3$ RIM RG series allowed [22] to restore convergence of the results obtained in the massive scheme. Herein, we apply this method to 5-loop functions, obtained in the minimal subtraction scheme.

This study focuses mainly on the convergence properties of the RIM RG series. In particular, we want to check whether the resummation procedure devised in the $d=0$ RIM in Ref. [27] and applied for the $d=3$ massive RG scheme in Ref. [22] is helpful for the $d=3$ RG functions in the minimal subtraction scheme. If so, its application will provide estimates for the RIM critical exponents as well.

The setup of this paper is organized as follows. First, the field-theoretical analysis of RIM critical behaviour is briefly reviewed, and expressions are given for the $\mathrm{RG}$ functions. The "subsequent resummation" procedure of Ref. [27] is then described, along with numerical data of the RIM critical behaviour provided as well. We end by giving conclusions and outlook.

\section{THE RESUMMATION AND THE RESULTS}

The critical behaviour of the RIM in the Euclidian space of $d=4-\varepsilon$ dimensions is governed by an effective Hamiltonian with two coupling constants [12], which can be obtained by making use of the replica method and taking the average over different configurations of quenched disorder:

$$
\begin{aligned}
\mathcal{H}(\varphi) & =\int \mathrm{d}^{d} R\left\{\frac{1}{2} \sum_{\alpha=1}^{n}\left[\left|\nabla \varphi_{\alpha}\right|^{2}+m_{0}^{2} \varphi_{\alpha}^{2}\right]\right. \\
& \left.+\frac{v_{0}}{4 !}\left(\sum_{\alpha=1}^{n} \varphi_{\alpha}^{2}\right)^{2}+\frac{u_{0}}{4 !} \sum_{\alpha=1}^{n} \varphi_{\alpha}^{4}\right\} .
\end{aligned}
$$

Here, $\varphi_{\alpha} \equiv \varphi_{\alpha}(R)$ is the $\alpha$ 's replica of a scalar field; $u_{0}>0$ and $m_{0}$ are the bare coupling constant of the pure model and the bare mass, $v_{0}<0$ is minus the variance of quenched disorder, and replica limit $n \rightarrow 0$ is implied.

To describe the long-distance properties of the model (2) near the second order phase transition point we use the field-theoretical RG method. In this approach, the change of the couplings $u, v$ under renormalization defines a flow in parametric space, governed by corresponding $\beta$-functions $\beta_{u}(u, v), \beta_{v}(u, v)$. The fixed points $u^{*}, v^{*}$ of this flow are given by solutions of the system of equations: $\beta_{u}\left(u^{*}, v^{*}\right)=0, \beta_{v}\left(u^{*}, v^{*}\right)=0$. The stable fixed point is defined as the fixed point where the stability matrix:

$$
B_{i j}=\frac{\partial \beta_{u_{i}}}{\partial u_{j}}
$$

possesses eigenvalues $\lambda_{i}$ with positive real parts. The accessible stable fixed point corresponds to the critical point of the system. At this point, the correlation length and susceptibility critical exponents $\nu$ and $\gamma$ are defined by the RG functions $\gamma_{2}(u, v)$ and $\gamma_{m}(u, v)$ via the relations:

$$
\nu^{-1}=2\left(1-\gamma_{m}\left(u^{*}, v^{*}\right)\right)
$$




$$
\gamma^{-1}=\frac{1-\gamma_{m}\left(u^{*}, v^{*}\right)}{1-\gamma_{2}\left(u^{*}, v^{*}\right)}
$$

Other critical exponents can be obtained from familiar scaling relations. For example, the hyperscaling relation allows us to express the specific heat critical exponent $\alpha$ in terms of space dimension $d$ and the correlation length critical exponent $\nu$ :

$$
\alpha=2-d \nu
$$

The results for the minimal subtraction scheme $R G$ functions corresponding to (2) in the replica limit $n \rightarrow 0$ can be derived in the 5-loop approximation from the appropriate expressions for RG functions of the $\varphi^{4}$-theory with $O(n)$-symmetric and cubic interactions obtained in the Ref. [26] and may be written as [25]:

$$
\begin{aligned}
\beta_{u} / u & =-\varepsilon+3 u+4 v-17 / 3 u^{2}-46 / 3 v u-82 / 9 v^{2}+32.54968284 u^{3}+123.1987313 v u^{2}+158.1816418 v^{2} u \\
& +60.32526811 v^{3}-271.6057842 u^{4}-1318.116311 v u^{3}-2452.429994 v^{2} u^{2}-2003.560971 v^{3} u \\
& -559.7143854 v^{4}+2848.568254 u^{5}+16789.89843 v u^{4}+40367.08593 v^{2} u^{3}+48971.12730 v^{3} u^{2} \\
& +29091.77179 v^{4} u+6377.751189 v^{5} \\
\beta_{v} / v & =-\varepsilon+8 / 3 v+2 u-14 / 3 v^{2}-22 / 3 v u-5 / 3 u^{2}+25.45714897 v^{3}+62.25499170 v^{2} u+36.36645522 v u^{2} \\
& +7 u^{3}-200.9263690 v^{4}-667.3761895 v^{3} u-650.5641816 v^{2} u^{2}-259.2586891 v u^{3}-39.91261012 u^{4} \\
& +2003.976188 v^{5}+8469.158907 v^{4} u+11721.60876 v^{3} u^{2}+7434.635066 v^{2} u^{3}+2344.277996 v u^{4} \\
& +301.5110976 u^{5}, \\
\gamma_{2} & =1 / 18 v^{2}+1 / 6 v u+1 / 12 u^{2}-1 / 27 v^{3}-1 / 6 v^{2} u-3 / 16 v u^{2}-1 / 16 u^{3}+125 / 648 v^{4}++125 / 108 v^{3} u \\
& +145 / 72 v^{2} u^{2}+65 / 48 v u^{3}+65 / 192 u^{4}-1.005978154 v^{5}-7.544836154 v^{4} u-18.04854621 v^{3} u^{2} \\
& -19.07838990 v^{2} u^{3}-9.627924878 v u^{4}-1.925584976 u^{5}, \\
& +686.375317 v^{3} u^{2}+737.493196 v^{2} u^{3}+376.1776339 v u^{4}+75.37777445 u^{5} . \\
& -1 / 3 v+1 / 2 u-5 / 18 v^{2}-5 / 6 v u-5 / 12 u^{2}+37 / 36 v^{3}+37 / 8 v^{2} u+251 / 48 v u^{2}+7 / 4 u^{3}-5.3808017 v^{4} \\
& -32.28481 v^{3} u-57.177011 v^{2} u^{2}-39.765731 v u^{3}-9.97815253 u^{4}+37.850485 v^{5}+283.878638 v^{4} u
\end{aligned}
$$

To obtain reliable quantitative results for the problem under consideration, an appropriate resummation procedure is implied. In Ref. [25] the Chisholm-Borel resummation technique was exploited for RG functions (7)-(10), which allowed obtaining a random fixed point $\left(u^{*}>0, v^{*}<0\right)$ increasing the order of approximation from 2-loop to 4-loop level. In the 5-loop order, however, the applied resummation technique does not lead to a real root for the random fixed point. It is not excluded that the absence of a fixed point solution might be connected with a possible Borel-nonsummability of the series under consideration. In this case, the 4-loop approximation is an "optimal truncation" for resummed RG functions in the minimal subtraction scheme.

Herein, we adopt another resummation technique, proposed recently in Ref. [27], where it was demonstrated analytically that the perturbation expansion for the free energy of the zero-dimensional quenched disordered Ising model is Borel summable, provided that the summation is carried out in two steps: first, in the strength of the original coupling $u$ of the pure Ising model and, subsequently, in the variance of quenched disorder, $v$. Such a method, implying the "unequality of rights" of the couplings $u, v$ was successfully used for $d=3$ RIM case in Ref. [22], where the 6-loop RG functions obtained in massive scheme were analyzed. The resummation procedure is as follows. Starting from the RG function of $p$-loop approximation in the form:

$$
f(u, v)=\sum_{k=0}^{p} \sum_{n=0}^{p-k} C_{n k} u^{n} v^{k}
$$

we rewrite it as a power series in $v$ : 


\section{BLAVATS'KA, YU. HOLOVATCH}

$$
f(u, v)=\sum_{k=0}^{p} A_{k}(u) v^{k}
$$

For each coefficient $A_{k}(u)$ we construct its Borel-Leroy image:

$$
A_{k}(u)=\sum_{n=0}^{p-k} C_{n k} u^{n} \Longrightarrow \sum_{n=0}^{p-k} \frac{C_{n k}(u t)^{n}}{\Gamma(n+j+1)}
$$

with $j$ as a fit parameter, then extrapolate (13) by rational Padé approximant [28]:

$$
\left[\frac{p-k-s_{k}}{s_{k}}\right](u t)
$$

and, finally, the resummed function $A_{k}^{\text {res }}(u)$ is obtained in the following form:

$$
A_{k}^{\mathrm{res}}(u)=\int_{0}^{\infty} \mathrm{d} t e^{-t} t^{j}\left[\frac{p-k-s_{k}}{s_{k}}\right](u t) .
$$

In our calculations, according to Ref. [22], we have fixed $s_{k}=1$ for all $0 \leq k \leq p$. Otherwise, most of the Padé approximants turn out to have poles on the positive real axis, so that integrals (15) do not exist.

The next step consists in substituting the computed coefficients $A_{k}^{\text {res }}(u)$ in (12). So, one obtains function (11) in the form of the power series in $v$ :

$$
\bar{f}(u, v)=\sum_{k=0}^{p} A_{k}^{\mathrm{res}}(u) v^{k}
$$

which now must be resummed in a similar way in the variable $v$. We introduce the parameter $q$ representing the number of terms in (16) which are to be resummed (note that resumming the functions in $u$ in the above we fixed $q=p$, as far as the summability of series in $u$ is established [22]). One of the problems arising here is an adequate choice of the parameter $q$. In Ref. [22] it was fixed to the value $q=p-1$, i.e., it was exploited the resummation of only the first $(p-1)$ terms of function (16), discarding the last, proportional to $v^{p}$. We, however, have tried 3 most natural possibilities, arising here:

- i) the same as proposed in [22];

- ii) fixing the value $q=p$, i.e., the resummation of the entire function $\bar{f}(u, v)$ which seems to be the most natural one;

- iii) fixing the value $q=p-1$, i.e., the resummation of $(p-1)$ first terms of function (16), finally adding the last term without resummation.

Performing the resummation we have only used the Padé approximants with linear denominators.

Applying the above method in order to obtain the random fixed point $\left(u^{*} \neq 0, v^{*} \neq 0\right)$, we resum the functions $\beta_{u}(u, v) / u$ and $\beta_{v}(u, v) / v$ and then look for a common zero with $\left(u^{*}>0, v^{*}<0\right)$.

First, we exploit the possibility (i) of resummation of the $\beta$-functions. Figure 1 depicts the lines of zeroes of the resummed $\beta$-functions in a 5 -loop approximation in the $u v$-plane. Intersections of the curves, i.e., the simultaneous zeroes of both $\beta$-functions correspond to the fixed points. In the region of couplings of interest one notes two physically accessible fixed points that are rather close to each other, one of which is unstable. The existence of a second physically accessible fixed point in the vicinity to the stable one decreases the reliability of results. Moreover, the bulk of studies of the RIM brings about the presence of only one fixed point in this region (see, e.g., $[3,18-21,25])$. Note, however, that a similar situation arises when the massive scheme RG functions are analyzed [22]. In the 4-loop approximation one obtains a more "natural" picture with one physically accessible fixed point:

$u^{*}=0.6890, v^{*}=-0.1440$ with $\lambda_{1}=0.254, \lambda_{2}=0.557$

similar to the one depicted in Fig. 2. The respective critical exponents read:

$$
\nu=0.665, \gamma=1.299, \alpha=0.005
$$

Note that we calculate the values of exponents $\nu, \gamma$ by means of substituting Eqs. (9), (10) into Eqs. (4), (5), applying the resummation procedure (11)-(16) to the resulting expansions and, finally, substituting the obtained values of stable fixed point coordinates in the derived expressions for critical exponents. The specific heat critical exponent $\alpha$ is then found using relation (6).

Applying the possibility (ii), in the 4-loop level one obtains a situation with 2 closely located random fixed points, as it is described above; the coordinates of stable one:

$u^{*}=0.6862, v^{*}=-0.1640$ with $\lambda_{1}=0.138, \lambda_{2}=0.711$

and corresponding critical exponents:

$$
\nu=0.662, \gamma=1.294, \alpha=0.014 \text {. }
$$

However, in the 5-loop level such a technique leads to an absence of a physically accessible stable fixed point. 


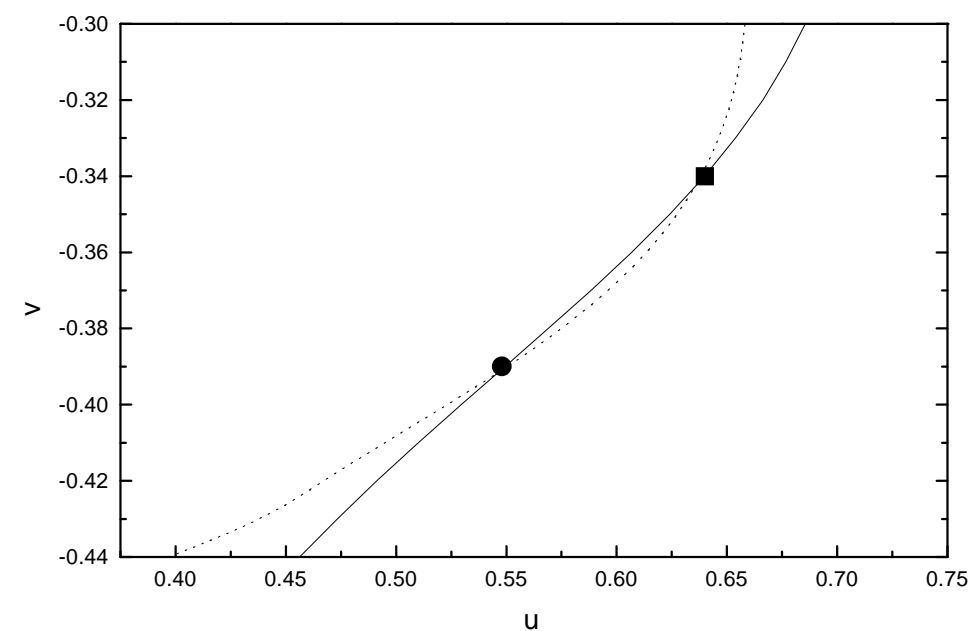

FIG. 1. The lines of zeroes of the resummed $\beta$-functions in 5-loop approximations. The way of resummation (i) is used (see the text). Solid line corresponds to $\beta_{v}=0$, dotted line depicts $\beta_{u}=0$. One can see the appearance of the 2 fixed points with $u^{*}>0, v^{*}<0$. Stable fixed point $u^{*}=0.6362, v^{*}=-0.3423$ is shown by a filled square, unstable one $\left(u^{*}=0.5450, v^{*}=-0.3922\right)$ is shown by a filled circle.

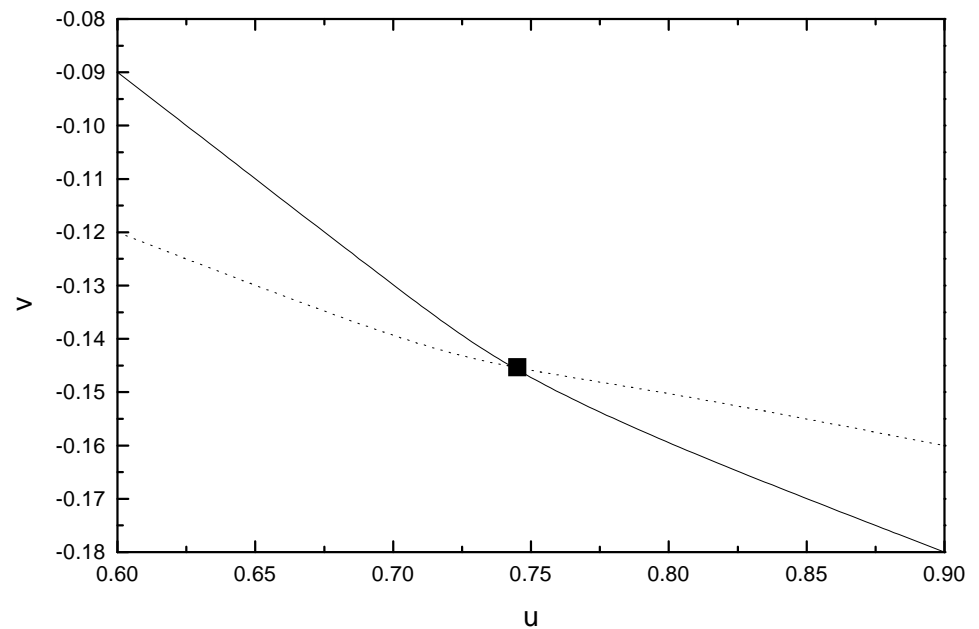

FIG. 2. The lines of zeroes of resummed $\beta$-functions in 5 -loop approximations. The way of resummation (iii) is used (see the text). Solid line corresponds to $\beta_{v}=0$, dotted line depicts $\beta_{u}=0$. Stable fixed point $u^{*}=0.7396, v^{*}=-0.1453$ is shown by a filled square.

The possibility (iii) turns out to be the most effective in our case. Its application leads to a stable fixed point, a topology of the $\beta$-functions intersection at which has a form similar to that in Fig. 2 and remains stable with increasing the order of approximation from 3-loop level to 5-loop one. A particular feature of the subsequent resummation procedure is that the topology of the intersections of the lines of zeroes of resummed $\beta$-functions essentially differs from that of the resummed $\beta$-functions in the lower orders of the perturbation theory (see, e.g., $[3,25])$. So, as we have already pointed above, exploiting the possibilities (i), (ii) of the subsequent resummation leads to the existence of an unstable fixed point with $\left(u^{*}>0, v^{*}<0\right)$ along with the stable one (such a situation arises in the massive scheme as well [22]). Moreover, new fixed points with $\left(u^{*} \neq 0, v^{*} \neq 0\right)$ emerge in a 


\section{BLAVATS'KA, YU. HOLOVATCH}

non-physical region $(u>0, v>0)$. This holds even if we have only one stable fixed point in the region of interest $(u>0, v<0)$. In Table 1 we present the values for the stable fixed points coordinates, critical exponents and stability matrix eigenvalues, obtained by means of method (iii). As was mentioned earlier, an application of this method leads to the only one stable fixed point in the physical region of couplings $(u>0, v<0)$.

\begin{tabular}{|c|cccccc|}
\hline Loop & $u^{*}$ & $v^{*}$ & $\nu$ & $\gamma$ & $\alpha$ & $\lambda_{1,2}$ \\
\hline 3 & 0.8276 & -0.1720 & 0.713 & 1.375 & -0.139 & $0.457 \pm 0.770 \mathrm{i}$ \\
4 & 0.6838 & -0.0907 & 0.672 & 1.296 & -0.016 & $0.357 \pm 0.456 \mathrm{i}$ \\
5 & 0.7396 & -0.1453 & 0.708 & 1.364 & -0.124 & $0.647 \pm 0.987 \mathrm{i}$ \\
\hline
\end{tabular}

Table 1. The resummed values of the stable fixed point coordinates and critical exponents of RIM. The way of resummation (iii) is used (see the text).

\section{CONCLUSIONS}

The main purpose of this study was to shed light on the convergence properties of the RIM RG expansions. Performing this analysis we obtained estimations for critical exponents as well (see Table 1). As was noticed in the Introduction, the values of the RIM critical exponents are estimated up to now in numerous studies in successive orders of perturbation theory. However, the very idea of the perturbation theory approach is based on the conjecture about the convergence of the results obtained. In the theory of critical phenomena, the RG perturbation expansions are proved to be Borel-summable (i.e., to give convergent results) only for the model with a simple $O(m)$-symmetry. The convergence still is not proved for RIM. Moreover, there exist serious indications [15] of the possible Borel-nonsummability of the expressions obtained. Therefore, the present RG estimations of the RIM critical exponents are based on the disputable assumption about their asymptotic nature. As long as relatively short perturbation theory series for the RIM RG functions were available, they provided rather convergent results [18-20]. Recently, the higher-order perturbation theory expansions have been obtained for the RIM [21,22], and the problem of convergence posed already in Refs. [15] emerged again. It was shown that in higher orders of perturbation theory an "optimal truncation" regime may occur, similarly to the situation in nonresummed asymptotic series. One encounters such a situation analyzing the 5-loop RG functions obtained on the basis of the minimal subtraction scheme where in 5-loop approximation no stable random fixed point was found using the Chisholm-Borel resummation technique [25]. On the other hand, the massive RG functions [21,22] resummed by the familiar Chisholm-Borel technique resulted in the presence of the stable random fixed point. However, the comparison of the data obtained in the successive orders of perturbation theory served as an indirect evidence of a possible non-asymptotic nature of the series [3]. Application of a subsequent resummation technique (see formulas (11)-(16)) [27] to the $d=3$ RIM RG functions in the massive scheme allowed [22] to restore convergence of the results obtained in the massive RG scheme. In our study, we applied the method of subsequent resummation to the RIM 5-loop RG functions obtained in the Ref. [26] on the basis of the minimal subtraction scheme. Indeed, it enabled us to get a stable picture of the presence of random fixed point in successive orders of the perturbation theory, including the 5loop approximation. However, the procedure turned out to be extremely sensitive to the choice of resummation parameters. It is the reason why we do not provide error bars to the numbers obtained. In spite of the fact that a more elaborated procedure of subsequent resummation allowed us to process the 5-loop RG series in the minimal subtraction scheme, still it is not excluded that the series under considerations, even resummed, are characterized by an "optimal truncation" behaviour. If this observation is valid it would serve as an evidence for Borel-nonsummability of the RIM RG functions.
[1] A. B. Harris, J. Phys. C 7, 1671 (1974).

[2] see, e.g: E. Brézin, J. C. Le Guillou, J. Zinn-Justin, in Phase Transitions and Critical Phenomena. Vol. 6, edited by C. Domb and M. S. Green, (Academic Press, London, 1976); D. J. Amit, Field Theory, the Renormalization Group, and Critical Phenomena (World Scien- tific, Singapore, 1989); J. Zinn-Justin, Quantum Field Theory and Critical Phenomena (Oxford University Press, 1996); H. Kleinert, V. Schulte-Frohlinde, Critical Properties of $\phi^{4}$-Theories (World Scientific, Singapore, 2001).

[3] R. Folk, Yu. Holovatch, T. Yavors'kii, preprint cond- 
mat/0106468 (2001); to appear in: Physics Uspiekhi (2002).

[4] G. H. Hardy, Divergent Series (Oxford, 1948).

[5] L. N. Lipatov, Zh. Eksp. Teor. Fiz. 72, 411 (1977).

[6] E. Brézin, J. Le Guillou, J. Zinn-Justin, Phys. Rev. D. 15, 1544 (1977).

[7] E. Brézin, G. Parisi, J. Stat. Phys. 19, 269 (1978).

[8] J.-P. Eckmann, J. Magnen, R. Sénéor, Commun. Math. Phys. 39, 251 (1975); J. S. Feldman, K. Osterwalder, Ann. Phys. 97, 80 (1976); J. Magnen, R. Sénéor, Commun. Math. Phys. 56, 237 (1977); J.-P. Eckmann, H. Epstein, Commun. Math. Phys. 68, 245 (1979).

[9] R. Guida, J. Zinn-Justin, J. Phys. A 31, 8103 (1998).

[10] D. E. Khmel'nitskii, Zh. Eksp. Teor. Fiz. 68, 1960 (1975) [JETP 41, 981 (1975)].

[11] T. C. Lubensky, Phys. Rev. B 11, 3573 (1975).

[12] G. Grinstein, A. Luther, Phys. Rev. B 13, 1329 (1976).

[13] C. Jayaprakash, H. J. Katz, Phys. Rev. B 16, 3987 (1977); B. N. Shalaev, Zh. Eksp. Teor. Fiz. 73, 2301 (1977) [Sov. Phys. JETP 46, 1204 (1977)].

[14] B. N. Shalaev, S. A. Antonenko, A. I. Sokolov, Phys. Lett. A 230, 105 (1997); R. Folk, Yu. Holovatch, and T. Yavors'kii, Pis'ma Zh. Eksp. Teor. Fiz. 69, 698 (1999) [Sov. Phys. JETP Letters 69, 747 (1999)].

[15] A. J. Bray, T. McCarthy, M. A. Moore, J. D. Reger, A. P. Young, Phys. Rev. B 36, 2212 (1987); A. J. McKa- ne, Phys. Rev. B 49, 12003 (1994).

[16] R. B. Griffiths, Phys. Rev. Lett. 23, 17 (1969).

[17] G. Parisi, in Proceedings of the Cargrése Summer School, 1973; G. Parisi, J. Stat. Phys. 23, 49 (1980).

[18] J. Jug, Phys. Rev. B 27, 609 (1983).

[19] I. O. Mayer, A. I. Sokolov, Fiz. Tverd. Tela 26, 3454 (1984) [Sov. Phys. Solid State 26, 2076 (1984)]; N. A. Shpot, Phys. Lett. A 142, 474 (1989).

[20] I. O. Mayer, A. I. Sokolov, B. N. Shalaev, Ferroelectrics 95, 93 (1989); I. O. Mayer, J. Phys. A 22, 2815 (1989).

[21] D. V. Pakhnin, A. I. Sokolov, Pis'ma Zh. Eksp. Teor. Fiz. 71, 600 (2000) [JETP Lett. 71, 412]; D. V. Pakhnin, A. I. Sokolov, Phys. Rev. B 61, 15130 (2000).

[22] A. Pelissetto, E. Vicari, Phys. Rev. B 62, 6393 (2000).

[23] G. t'Hooft, M. Veltman, Nucl. Phys. B 44, 189 (1972).

[24] R. Schloms, V. Dohm, Europhys. Lett. 3, 413 (1987); R. Schloms, V. Dohm, Nucl. Phys. B 328639 (1989).

[25] R. Folk, Yu. Holovatch, T. Yavorskii, Phys. Rev. B 61, 15114 (2000).

[26] H. Kleinert, V. Schulte-Frohlinde, Phys. Lett. B 342, 284 (1995).

[27] G. Alvarez, V. Martín-Mayor, and J. J. Ruiz-Lorenzo, J. Phys. A 33, 841 (2000).

[28] G. A. Baker, P. Graves-Morris Padé Approximants (Assison-Wesley, Reading, Mass., 1981).

\title{
ТРИВІМИРНА ВИПАДКОВА МОДЕЛЬ ІЗИНГА: ПЕРЕСУМОВУВАННЯ П'ЯТИПЕТЛЕВИХ РЯДІВ
}

\author{
В. Блавацька ${ }^{1}$, Ю. Головач ${ }^{1,2}$ \\ ${ }^{1}$ Інститут фізики конденсованих систем НАН Украйни \\ вул. Свенціцького, 1, Львів, 79011, Украӥна \\ 2 Львівський начіональний університет імені Івана Франка, кафедра теоретичной фізики \\ вул. Драгоманова, 12, Львів, 79005, Украйна \\ E-mail:viktoria@icmp.lviv.ua,hol@icmp.lviv.ua
}

\begin{abstract}
Досліджено критичну поведінку $d=3$-вимірної моделі Ізинга зі слабким замороженим безладом шляхом аналізу при $d=3$ рядів для ренормгрупових функщій, отриманих у схемі мінімального віднімання. Недавно було встановлено [R. Folk, Yu. Holovatch, T. Yavors'kii, Phys. Rev. B 61, 15114 (2000)], що пересумованим ренормгруповим функщіям властива поведінка "оптимального обрізання", якщо застосувати техніку пересумовування Чізгольма-Бореля. Це привело до припущення щодо остаточности 4-петлевого наближення для ренормгрупових функцій розведеної моделі Ізинга в $d=3$ схемі мінімального віднімання. Ми застосовуємо метод послідовного пересумовування, розвинутий у контексті $d=0$-вимірної розведеної моделі Ізинга, обговорюємо властивості збіжности рядів і подаємо результати для критичних показників, збільшуючи порядок наближення до 5-петлевого рівня.
\end{abstract}

